\title{
Erratum to: Impact of radioguided occult lesion localization on the correct excision of malignant breast lesions: effect of histology and tumor size
}

\author{
John Patrick Pilkington Woll - Montserrat Cortés Romera • Ana María García Vicente • \\ Beatriz González García • Margarita Delgado Portela • Jose Manuel Cordero García • \\ Ricardo Pardo García · Ceferino Molino Trinidad · Angel María Soriano Castrejón
}

Published online: 6 April 2011

(C) The Japanese Society of Nuclear Medicine 2011

\section{Erratum to: Ann Nucl Med}

DOI 10.1007/s12149-010-0453-z

Errors occurred in designating the authors' forenames and surnames. The names of the authors should be shown as follows:

Surname: Pilkington Woll

Forename: John Patrick

Surname: Cortés Romera

Forename: Montserrat

Surname: García Vicente

Forename: Ana María
Surname: González García

Forename: Beatriz

Surname: Delgado Portela

Forename: Margarita

Surname: Cordero García

Forename: Jose Manuel

Surname: Pardo García

Forename: Ricardo

Surname: Molino Trinidad

Forename: Ceferino

Surname: Soriano Castrejón

Forename: Angel María

The online version of the original article can be found under doi:10.1007/s12149-010-0453-z.

J. P. Pilkington Woll $(\varangle) \cdot$ A. M. García Vicente .

B. González García · J. M. Cordero García .

A. M. Soriano Castrejón

Nuclear Medicine Department, University General Hospital,

Obispo Rafael Torrijas s/n, 13005 Ciudad Real, Spain

e-mail: patrick_pilkington@yahoo.com

M. Cortés Romera

PET Unit, Centre IDI Bellvitge, Bellvitge University Hospital,

L'Hospitalet de Llobregat, Barcelona, Spain

M. Delgado Portela

Pathology Department, University General Hospital,

Obispo Rafael Torrijas s/n, 13005 Ciudad Real, Spain

R. Pardo García

Surgery Department, University General Hospital,

Obispo Rafael Torrijas s/n, 13005 Ciudad Real, Spain

C. Molino Trinidad

Radiology Department, University General Hospital,

Obispo Rafael Torrijas s/n, 13005 Ciudad Real, Spain 